\title{
Detection of neuronal activity and metabolism in a model of dehydration-induced anorexia in rats at 14.1 T using manganese-enhanced MRI and ${ }^{1} H$ MRS
}

\author{
Nathalie Just ${ }^{a, b *}$ and Rolf Gruetter ${ }^{a, b, c}$
}

\begin{abstract}
In this study, hypothalamic activation was performed by dehydration-induced anorexia (DIA) and overnight food suppression (OFS) in female rats. The assessment of the hypothalamic response to these challenges by manganeseenhanced MRI showed increased neuronal activity in the paraventricular nuclei (PVN) and lateral hypothalamus (LH), both known to be areas involved in the regulation of food intake. The effects of DIA and OFS were compared by generating $T$-score maps. Increased neuronal activation was detected in the PVN and LH of DIA rats relative to OFS rats. In addition, the neurochemical profile of the PVN and LH were measured by ${ }^{1} \mathrm{H}$ MRS at $14.1 \mathrm{~T}$. Significant increases in metabolite levels were measured in DIA and OFS relative to control rats. Statistically significant increases in $\gamma$-aminobutyric acid were found in DIA $(p=0.0007)$ and OFS $(p<0.001)$ relative to control rats. Lactate increased significantly in DIA $(p=0.03)$, but not in OFS, rats. This work shows that manganese-enhanced MRI coupled to ${ }^{1} \mathrm{H}$ MRS at high field is a promising noninvasive method for the investigation of the neural pathways and mechanisms involved in the control of food intake, in the autonomic and endocrine control of energy metabolism and in the regulation of body weight. Copyright $\odot 2011$ John Wiley \& Sons, Ltd.
\end{abstract}

Keywords: MEMRI; Dehydration Induced Anorexia; hypothalamus; $14.1 \mathrm{~T}$

\section{INTRODUCTION}

Recently, manganese-enhanced MRI (MEMRI) has been demonstrated to show an increased potential for the visualization of functional neural circuits and anatomy in the brain of rodents $(1,2)$. In vivo tract tracing MEMRI methods enable the study of the topography, plasticity and function of neuronal circuits in individual subjects $(3,4)$, whereas activation-induced MEMRI methods enable the mapping of brain activation independent of hemodynamic changes and following cellular depolarization $(1,2,5)$. Compared with blood oxygen level-dependent functional MRI, MEMRI methods have a high sensitivity and signal-to-noise ratio (SNR), allow high spatial resolution three-dimensional MRI, can reveal cortical layers and can be helpful for connectivity studies (6).

Whether MEMRI techniques can provide surrogate markers of neuronal activity remains to be answered, as the contrast mechanisms following manganese injection depend on multiple factors ranging from the type of functional challenge to the specific type and number of neurons activated $(2,5)$.

An important amount of attention is currently being focused on obtaining an understanding of the neural mechanisms of feeding $(7,8)$. In order to understand the organization, interaction and function of the neuronal pathways controlling feeding in normal, obese and anorexic states, the identification of important neurons and their connections is essential.

Anorexia is characterized by abnormal patterns of feeding behavior and severe weight loss (9). The appetite-regulating network resides in the hypothalamus, where food intake is controlled through pathways emanating from the arcuate nucleus

\footnotetext{
* Correspondence to: N. Just, Laboratory for Functional and Metabolic Imaging (LIFMET), Centre d'Imagerie Biomédicale (CIBM), CH-1015 Lausanne, Switzerland.

E-mail: nathalie.just@epfl.ch

a N. Just, R. Gruetter

Laboratory for Functional and Metabolic Imaging, Ecole Polytechnique Fédérale de Lausanne, Lausanne, Switzerland

b N. Just, R. Gruetter

Department of Radiology, University of Lausanne, Lausanne, Switzerland

C R. Gruetter

Department of Radiology, University of Geneva, Geneva, Switzerland
}

Abbreviations used: $A M P$, adenosine-5'-monophosphate-activated kinase; $A R C$, arcuate nucleus; AgRP, Agouti-related protein; $\mathrm{Cr}$, creatine; $\mathrm{CRH}$, corticotropin-releasing hormone; CTL, control; DIA, dehydration-induced anorexia; FASTMAP, fast automatic shimming technique by mapping along projections; GABA, $\gamma$-aminobutyric acid; Gln, glutamine; GLP1, glucagon-like peptide 1; Glu, glutamate; HR-MAS, High Resolution magic angle spinning; Lac, lactate; LH, lateral hypothalamus; MEMRI, manganese-enhanced MRl; myo-Ins, myo-inositol; NAA, N-acetylaspartate; NPY, neuropeptide $Y$; OFS, overnight food suppression; PaAP, anterior parvicellular part of the paraventricular nucleus; PALM, anterior magnocellular part of the paraventricular nucleus of the hypothalamus; PaMP, medial parvicellular zone of the paraventricular nucleus of the hypothalamus; POMC/CART, pro-opiomelanocortin/cocaine-amphetamineregulated transcript; PaPo, posterior part of the paraventricular nucleus of the hypothalamus; PaV, ventral part of the paraventricular nucleus of the hypothalamus; $P C r$, phosphocreatine; $P I T$, pituitary gland; $P V N$, paraventricular nucleus/nuclei; ROI, region of interest; SNR, signal-to-noise ratio; SPECIAL, spin echo, full intensity acquired localized; Tau, taurine; TRH, thyrotropin-releasing hormone; VAPOR, variable pulse power and optimized relaxation delays; $3 \mathrm{~V}$, third ventricle. 
(ARC) and terminating in the paraventricular nucleus (PVN) (10). These findings led to the discovery of additional circuits, such as the anorexigenic pathway emanating in the ARC and the modulatory pathway emanating in the lateral hypothalamus (LH) and the ventromedial hypothalamus. These pathways are functionally modulated by neuropeptide $Y$ (NPY), the appetitestimulating neurotransmitter in the brain. Experimental findings have revealed that NPY secretion is increased in the PVN in association with increased hunger produced by fasting and dieting (10). Within the ARC-PVN, homeostasis is deregulated by the disruption of the NPY network, whether low or high NPY abundance is elicited at target sites (10).

In this study, MEMRI was used to investigate the underlying mechanisms of anorexia using a model of dehydration-induced anorexia (DIA) in female rats (11). This model of anorexia has been studied extensively (11-14) and presents reproducible characteristics that are useful to test the ability of MEMRI techniques to investigate neuronal activity and neuronal pathways controlling feeding behavior. In addition to MEMRI, the neurochemical profile of PVN-LH was determined using ${ }^{1} \mathrm{H}$ MRS at $14.1 \mathrm{~T}$.

\section{MATERIALS AND METHODS}

\section{Animal preparation}

All studies were performed in accordance with the local ethics committee and met the guidelines of the appropriate government agency. Thirty-six female Wistar rats [225-250 g body weight on Day 0 (D0)] were divided into three groups: control $(C T L, n=12)$, overnight food suppression (OFS, $n=8$ ) and dehydration-induced anorexia (DIA, $n=16)$ groups. Two rats per group were discarded from the study as failure of $\mathrm{MnCl}_{2}$ infusion was suspected. MR experiments were conducted on one rat per group per session. Animals were acclimatized in an animal facility under humidity and temperature-controlled conditions on a 12-h light/dark cycle during 1 week before the beginning of the experiments. Animals were allowed access to standard chow and drinking water ad libitum during this period.

On D0, animals were placed in individual cages. Animals from the CTL and OFS groups received water and food ad libitum; the DIA group received $2.5 \% \mathrm{NaCl}$ (Sigma, Zofingen, Switzerland) as drinking solution (15). On D3 at $18.00 \mathrm{~h}$, the OFS rats had their chow removed until the MR scan was performed, $24 \mathrm{~h}$ later. The amounts of food and drink were measured at $18.00 \mathrm{~h}$ on D0, D1, D3, D7, D10 and D14. The body weights of the rats were also measured. On D3, rats from each group were anesthetized with $2 \%$ isoflurane in a mixture of $\mathrm{O}_{2}$ and $\mathrm{N}_{2} \mathrm{O}$ via a facemask. The tail vein was catheterized for the intravenous infusion of $\mathrm{MnCl}_{2}$. Each rat received an intravenous infusion of $100 \mathrm{mg} / \mathrm{kg}$ at $100 \mathrm{mM} \mathrm{MnCl} 2$ at a rate of $0.5-1 \mathrm{~mL} / \mathrm{h}$ via the tail vein to avoid cardiac arrest. Animals were gently manipulated all the time to avoid stress and suffering. During infusion and until animals were completely recovered from anesthesia, their temperature was carefully monitored and kept at $37 \pm 1{ }^{\circ} \mathrm{C}$.

For ${ }^{1} \mathrm{H}$ MRS studies, five female Wistar rats per group were used. These rats did not undergo $\mathrm{MnCl}_{2}$ infusions and were scanned on D4 after the start of the anorexic protocol (D0) for the DIA group of rats.

During the MR experiments, rats were anesthetized with $2 \%$ isoflurane in a mixture of $\mathrm{O}_{2}$ and $\mathrm{N}_{2} \mathrm{O}$. The rats were positioned in a dedicated holder with head fixation. Their body temperature was maintained at $37.5 \pm 0.5^{\circ} \mathrm{C}$ using a temperature-controlled water circulation and a rectal probe. Their respiration rate was monitored throughout the experiment. MRI was performed on D4, 24h after $\mathrm{MnCl}_{2}$ infusion.

\section{MRI data acquisition}

All the experiments were performed on a 14.1-T/26-cm horizontal bore magnet (Magnex Scientific, Oxford, UK). The magnet was equipped with $12-\mathrm{cm}$ inner diameter actively shielded gradient sets (Magnex Scientific) allowing a maximum gradient of $400 \mathrm{mT} / \mathrm{m}$ in $120 \mu \mathrm{s}$. A home-built quadrature $17-\mathrm{mm}$ transmit-receive surface coil was used. Field homogeneities were adjusted using an EPI version of FASTMAP (fast automatic shimming technique by mapping along projections) (16-18).

After the preliminary adjustments (tuning, shimming, acquisition of scout images for accurate positioning), fast spin echo images $\left(\mathrm{TR} / \mathrm{TE}=2500 / 12 \mathrm{~ms}\right.$; eight echoes; field of view, $25 \times 25 \mathrm{~mm}^{2}$; matrix size, $256 \times 256$; axial slices; slice thickness, $0.5 \mathrm{~mm}$; eight averages) were acquired. Three-dimensional gradient echo $T_{1}$-weighted images (TR/TE $=20 / 5 \mathrm{~ms}$; flip angle, $70^{\circ}$ field of view, $25 \times 25 \times 25 \mathrm{~mm}^{3}$; matrix size, $256 \times 256 \times 128$; coronal slices; bandwidth, $25 \mathrm{kHz}$; five averages) were acquired as part of the MEMRI protocol.

\section{Image analysis}

MR images were processed and analyzed using Image processing software (Image J 1.3.1_13; National Institutes of Health, Bethesda, MD, USA) as well as custom-written routines running in Matlab (Matlab 7.4; The MathWorks Inc., Natick, MA, USA). The signal intensity of each voxel of the three-dimensional images was normalized by the signal in a reference area to minimize variations caused by system instability.

\section{Bias field correction}

Radiofrequency inhomogeneities over three-dimensional images were corrected using an in-house bias field algorithm $(19,20)$ inspired by Styner et al. (21). The bias field 'corrupted' signal intensity was modeled using $I_{\text {measured }}(y)=I_{\text {true }}(y) \times \beta(y)+N(y)$, where $I$ is the signal intensity, $\beta$ is the multiplicative bias field, $N$ is the noise and $y$ is the pixel position. Legendre polynomials were used to model the bias field in combination with a priori knowledge of the shape of the object to be corrected and the estimation of tissue classes using a manually defined mask. The segmentation of rat brain images was based on a threedimensional atlas (UCLA rat brains; www.loni.ucla.edu/). The registration process was performed using the similarity threedimensional transform and the 'thin plate spline' transform after selection of several landmarks on both source (atlas) and target images. Estimation of tissue class statistics used a nonlinear optimization evolutionary algorithm. Initial class values must be given to the algorithm for parameter recognition and were based on the histograms determined from manually drawn masks on images to be corrected. The adequate determination of the bias field and of the classes depends on the appropriate choice of the degree of the Legendre polynomials, the number of iterations and the number of classes chosen $(19,20)$. All the images obtained in the present study were bias field corrected.

\section{Calculation of SNR}

Regions of interest (ROIs) were drawn in the ARC, PVN, LH, pituitary gland (PIT), hippocampus and amygdala with reference to the atlas 
of Paxinos and Watson (22). For each rat, SNR was calculated in these ROls, as well as on a pixel-by-pixel basis. An ROI was drawn in the image background for noise measurement. The positions of these 'noise' ROls were randomized over each group of animals. The ratio of the signal intensity to the noise standard deviation was calculated.

\section{Group averaging}

For a better definition of the differences between CTL, OFS and DIA rats, and in order to target specific neuronal activation following manganese enhancement in the hypothalamus, threedimensional, gradient echo, $T_{1}$-weighted images from rats that had underdone MRI were compared. Single images from CTL, OFS and DIA rats from the same plane were adjusted to the corresponding plane of the rat atlas of Paxinos and Watson (22). To generate an averaged $\mathrm{MnCl}_{2}$ enhancement map from animals in the same group and to compare them, threedimensional images corresponding to the same plane were coregistered to the corresponding plane of a chosen rat per group. A group $\mathrm{MnCl}_{2}$ enhancement map was calculated by averaging individual three-dimensional MR images in each group. In addition, standard deviation maps were generated.

\section{Comparison of $\mathrm{MnCl}_{2}$ enhancement maps}

To compare the enhancement patterns of the different feeding behaviors, a $T$-score was computed using:

$$
T=\frac{\bar{x}-\bar{y}}{\sqrt{\frac{\mathrm{SD}_{x}^{2}}{n}+\frac{\mathrm{SD}_{y}^{2}}{m}}}
$$

where $\bar{x}$ and $\bar{y}$ are the respective averaged signal intensities of the nearest four pixels of each pixel within the two group-averaged enhancement maps compared. $\mathrm{SD}_{x}{ }^{2}$ and $\mathrm{SD}_{y}{ }^{2}$ are the respective variances within the two group-averaged enhancement maps compared. $n$ and $m$ represent the respective number of elements. The $T$-scores were thus computed to compare the similarity between the enhancement patterns of the corresponding clusters in the LH and PVN of the CTL, OFS and DIA group-averaged enhancement maps. The $T$-scores were computed successively to compare CTL and OFS, CTL and DIA and, finally, OFS and DIA groups.

\section{Statistical analysis}

To investigate the enhancement patterns of the different food regimes, $\mathrm{MnCl}_{2}$ enhancement maps were compared using Student's $t$-test. The enhancement maps were thresholded at $p<0.05$ (one-tailed, uncorrected for multiple comparisons).

\section{${ }^{1} \mathrm{H}$ MRS in the hypothalamus}

Localized ${ }^{1} \mathrm{H}$ spectroscopy was performed using the SPECIAL (spin echo, full intensity acquired localized) technique (23), employing $\mathrm{TE} / \mathrm{TR}=2.8 / 4000 \mathrm{~ms}$ in combination with outer volume suppression and VAPOR (variable pulse power and optimized relaxation delays) water suppression (24). A voxel of interest of $50 \mu \mathrm{L}$ was localized in the PVN-LH region for all the rats using the previously acquired three-dimensional gradient echo images. All first- and second-order shims were re-adjusted using FASTMAP (17) in the voxel of interest, which resulted in water linewidths of $23 \pm 5 \mathrm{~Hz}$. To obtain adequate SNR, 640 scans were acquired. For absolute quantification, the water signal was acquired using identical parameters, except without water suppression and averaging eight scans.

\section{Quantification and statistics}

The in vivo ${ }^{1} \mathrm{H}$ MR spectra were processed using LCmodel (25). In this study, 18 metabolite concentrations were quantified using databases of simulated spectra of metabolites $(24,25)$.

All data are presented as the mean \pm standard error of the mean (SEM). Unpaired Student's $t$-tests were applied to compare data acquired in the three groups of rats.

\section{RESULTS}

\section{Effect of DIA on body weight, food and drink intake}

The body weight changes over 14 days for CTL, DIA and OFS rats are presented in Fig. 1A. DIA rats demonstrated a significant weight loss compared with CTL and OFS rats ( $t$-test, $p<0.0001)$ on each measurement day. A significant body weight loss was also measured for DIA rats relative to their body weight on D0 ( $t$-test, $p<0.0001$ ).

Over 14 days, CTL and OFS rats had regular and continuously increasing food and drink intakes (Fig. 1B). The food diet was significantly reduced for DIA rats compared with the other groups. The drink intake increased for the DIA group from D4 and was significantly higher than that for the CTL group at D14 $(p<0.05)$ (Fig. 1C).

\section{Detection of neuronal responses in the hypothalamus by MEMRI}

MEMRI was performed on $\mathrm{D} 4,24 \mathrm{~h}$ following $\mathrm{MnCl}_{2}$ intravenous injection into the tail vein for each rat. Three-dimensional, gradient echo, $T_{1}$-weighted images acquired at $14.1 \mathrm{~T}$ of CTL, DIA and OFS rats are shown in Fig. $2 \mathrm{~A}$. At $24 \mathrm{~h}$ post $-\mathrm{MnCl}_{2}$ injection, characteristic MRI signal enhancement was noticed in various brain regions for all rats independent of their diet condition: the olfactory bulb, hippocampus, ventricles, hypothalamus, PIT and cerebellum $(n=30)$. The hypothalamus was the main focus of the present study. Figure $2 \mathrm{~B}$ shows a schematic overview of the hypothalamic regions involved in the control of appetite. Some of these regions showed more specific enhancement in DIA and OFS rats, as illustrated in Fig. 2C, where hypothalamic ROls as outlined in Fig. $2 \mathrm{~A}$ are shown for CTL, DIA and OFS rats.

Figure 3A compares the mean SNR for each group of rats in the LH, PVN, ARC, PIT, hippocampus and amygdala. A significant mean SNR increase was detected in LH $(p<0.003), \mathrm{PVN}(p<0.0003)$ and ARC $(p=0.02)$ in DIA rats with respect to CTL rats. OFS rats presented increased mean SNR values identical to DIA in all anatomical structures investigated except for ARC, but comparisons with CTL rats did not reach statistical significance ( $p>0.05)$.

Examples of SNR pixel-by-pixel maps in individual rats examined on the same day (D4), 4 days after the onset of the hypertonic saline treatment, are shown in Fig. 3B, C. A rectangular $\mathrm{ROI}$ was drawn encompassing PVN and $\mathrm{LH}$ at the same slice level for CTL, DIA and OFS rats. Figure 3D depicts a schematic drawing of coronal slices from the atlas of Paxinos and Watson (22), and corresponding to the slices shown in Fig. 3B, C. The SNR level was significantly higher in PVN and LH of DIA rats than in CTL and OFS rats. The identification of specific structures, such as the medial parvicellular zone of the PVN of the hypothalamus (PaMP), anterior magnocellular part of the 

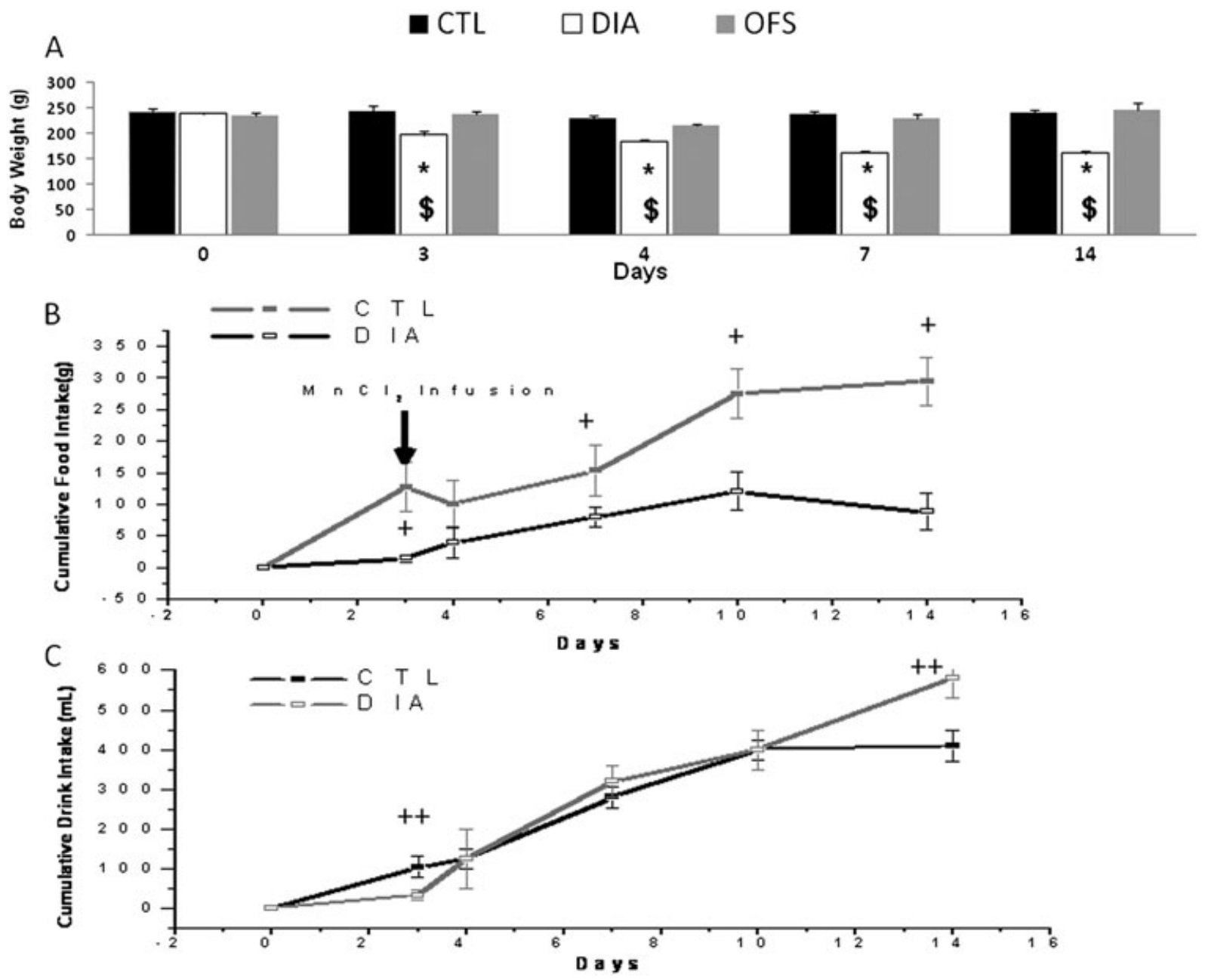

Figure 1. Body weight, cumulative food intake and cumulative liquid intake measurements over 14 days. (A) Body weight records (CTL, control; DIA, dehydration-induced anorexia; OFS, overnight food suppression). (B) Food consumed by CTL and DIA female rats receiving food ad libitum. (C) Liquid intake. CTL and OFS rats consumed tap water; DIA rats received a $2.5 \% \mathrm{NaCl}$ solution. All values are the means $(\mathrm{g}, \mathrm{mL}) \pm \mathrm{standard}$ error of the mean (SEM) per group of rats. Student's $t$-test showed significant differences: ${ }^{*}, \$,+,++, p<0.05$.

PVN of the hypothalamus (PALM), and ventral (PaV) and posterior (PaPo) parts of the PVN of the hypothalamus (Fig. 3D), is difficult, but higher SNR values can readily be noticed along the third ventricle (3V) in DIA relative to CTL and OFS SNR maps.

\section{Comparison of the neuronal responses to CTL, OFS and DIA food regimes in the PVN and LH}

The $T$-score color-coded maps are shown (Fig. 4A-C) for the comparisons OFS-CTL (Fig. 4A), DIA-CTL (Fig. 4B) and DIA-OFS (Fig. 4C). Several enhancing structures were identified by $T$-scores. Low $T$-scores ( $T$-score $<15, p \geq 0.05$ ) were found in all the hypothalamic structures in the DIA-OFS $T$-score maps. However, DIA-OFS T-score maps demonstrated that small differences existed between DIA and OFS rats. DIA animals showed higher hypothalamic activation than OFS rats. The greatest differences $(10<T$-score $<15)$ were located along $3 \mathrm{~V}$ at the level of PVN. Statistically significant differences were found over the entire brain slice for OFS-CTL and DIA-CTL T-score maps $(15<T$-score $<30, p<0.005)$. The $T$-scores were highest in the hypothalamus, in the nuclei of the septal complex and in the amygdala $(20<T$-score $<30)$ in DIA versus CTL rats. The $T$-scores were also particularly elevated in OFS versus CTL rats with differences covering much of the brain slice $(15<T$-score $<30)$. Within the hypothalamic regions, $T$-score maps revealed important differences between DIA and OFS rats. In particular, higher $T$-scores were readily noticed in $\mathrm{LH}$ in DIA relative to CTL rats compared with OFS relative to CTL rats. Moreover, the highest $T$-scores were found above the fornix for DIA-CTL $(T$-score $\sim 30)$ and OFS-CTL $(20<T$-score $<30) T$-score maps in areas corresponding to PaMP and PaPo. In DIA-CTL T-score maps, high $T$-scores were also found in the central part of the anterior hypothalamic area or the posterior zone of the LH in the subfornical area, whereas, in OFS-CTL T-score maps, high $T$-scores were located in areas under the PVN corresponding to the posterior part of the anterior hypothalamic area.

\section{Comparison of the neurochemical profiles of PVN at 14.1 T in DIA, OFS and CTL rats}

Examples of single-voxel spectra measured by the SPECIAL technique with peak assignment successively in CTL, OFS and DIA rats on D4 are shown in Fig. 5B, with the voxels of interest drawn in Fig. 5A.

Figure $5 \mathrm{C}$ compares the average metabolite concentrations in the PVN-LH region for each group of rats. Larger metabolite 


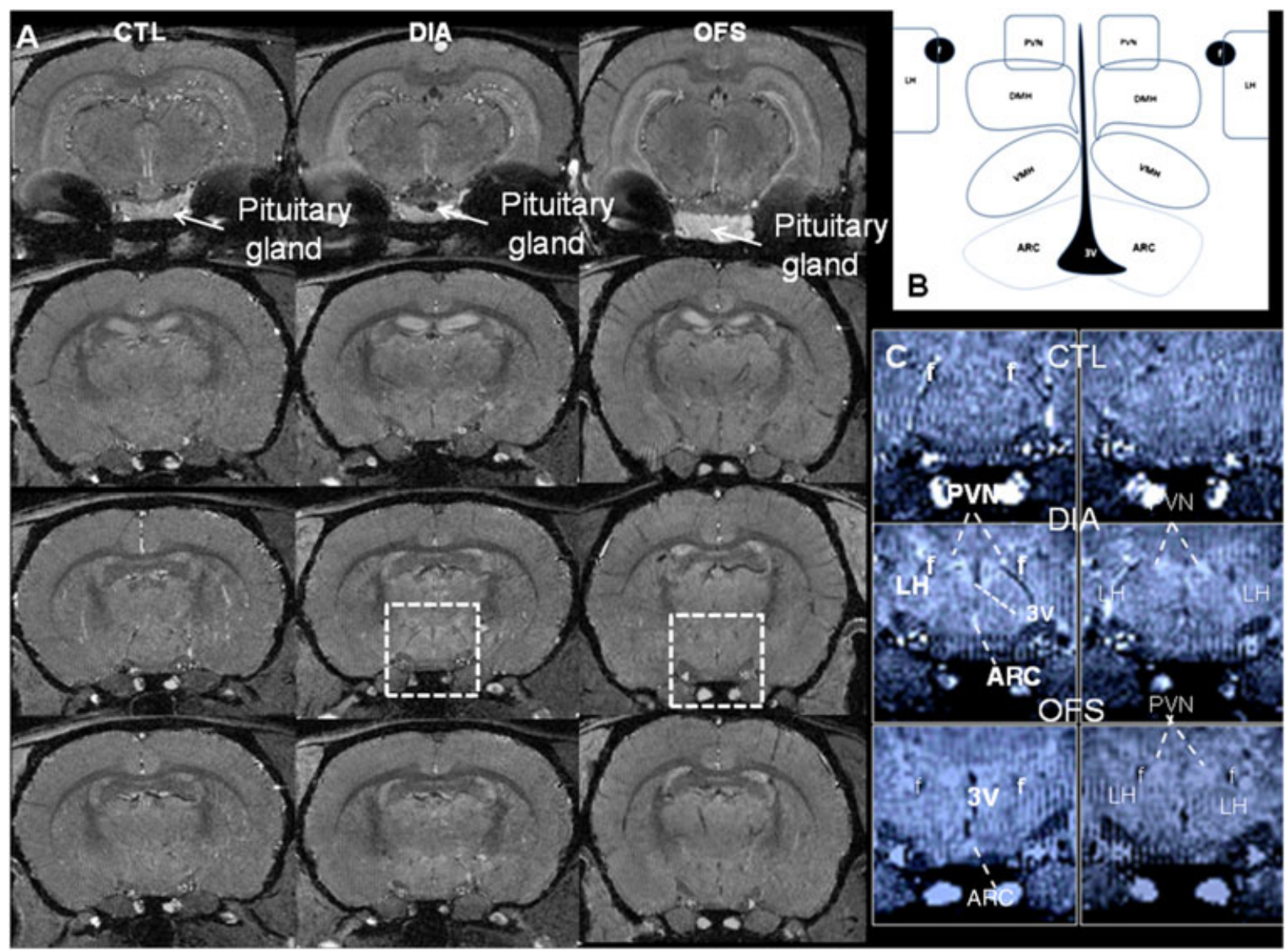

Figure 2. Examples of bias field-corrected, three-dimensional, $T_{1}$-weighted images acquired at $14.1 \mathrm{~T}$ in individual rats scanned on the same day at $24 \mathrm{~h}$ post $-\mathrm{MnCl}_{2}$ infusion (TR/TE $=20 / 5 \mathrm{~ms}$; flip angle, $70^{\circ}$; field of view, $25 \times 25 \times 25 \mathrm{~mm}^{3}$; matrix size, $256 \times 256 \times 128$; coronal slices; bandwidth, $25 \mathrm{kHz}$ ). (A) CTL, control; DIA, dehydration-induced anorexia; OFS, overnight food suppression. Images are shown at the same level for comparison. The square regions of interest (ROls) encompassing the hypothalamus are shown. Zoomed images of these ROls are shown in (C). (B) Schematic overview of the hypothalamic structures involved in the control of appetite. ARC, arcuate nucleus; DMH, dorsomedial hypothalamic nucleus; $f$, fornix; LH, lateral hypothalamus; PVN, paraventricular nucleus; VMH, ventromedial hypothalamic nucleus; 3V, third ventricle. (C) Hypothalamic structures from (A) in CTL, DIA and OFS rats. PVN, LH and ARC structures show enhancement in DIA and OFS rats relative to CTL rats.

concentrations were measured for OFS and DIA rats than for CTL rats. Significant increases were found for $\gamma$-aminobutyric acid (GABA) (unpaired $t$-test, two-tailed: CTL versus DIA, $p=0.0007 ; \mathrm{CTL}$ versus OFS, $p=0.0024$ ), glutamate (Glu) (unpaired $t$-test, twotailed: CTL versus OFS, $p=0.0005)$, myo-inositol (myo-Ins) ( $t$-test: CTL versus DIA, $p=0.008$ ), taurine (Tau) (unpaired $t$-test, twotailed: CTL versus DIA, $p<0.05$; CTL versus OFS, $p<0.03$ ), lactate (Lac) (unpaired $t$-test, two-tailed: CTL versus DIA, $p=0.035$ ), $\mathrm{N}$-acetylaspartate (NAA) (unpaired $t$-test, two-tailed: CTL versus OFS, $p=0.0014)$, creatine $(\mathrm{Cr})$ (unpaired $t$-test, two-tailed: $C T L$ versus DIA, $p=0.035$ ) and phosphocreatine ( $\mathrm{PCr}$ ) (unpaired $t$-test, two-tailed: CTL versus OFS, $p=0.021)$. The DIA and OFS groups of rats showed no statistically significant differences for the metabolite concentrations in the present study, except for Glu $(p<0.05)$ and NAA $(p=0.018)$. With the hypothesis that DIA induced a $10 \%$ loss of water, the metabolic profile of the DIA rate was corrected (DIA-corrected) and is also represented in Fig 5C. Only GABA ( $t$-test, CTL versus DIA-corrected, $p=0.0008$ ), myo-Ins (unpaired $t$-test, two-tailed: CTL versus DIA-corrected, $p=0.011$ ) and Lac (unpaired $t$-test, two-tailed: CTL versus DIAcorrected, $p<0.05)$ conserved significantly higher levels for DIA relative to $C T L$ rats.

\section{DISCUSSION}

In this study, we have shown that $\mathrm{MnCl}_{2}$ can be used to detect differences in the hypothalamus of female rats under different feeding regimes. These results suggest that MEMRI is a promising noninvasive method for the investigation of the neuronal activation and neuronal circuitry involved in the autonomic nervous system. Many studies have been conducted to obtain an understanding of the mechanisms of DIA (11-14). DIA rats limit their spontaneous food intake despite the presence of endocrine and neuropeptidergic profiles of negative energy balance, and share an identical profile of caloric deficit markers with food-restricted animals (11). Unlike food-restricted animals, spontaneous food intake in DIA animals (11) is not stimulated by undetectable levels of circulating leptin and insulin, increased plasma glucocorticoid concentrations, elevated NPY gene expression in the ARC and body weight loss. Dehydration stimulates the compensatory drinking of water, which subsequently helps to restore both intracellular and extracellular fluid compartments (26). These adaptive processes and responses to osmotic dehydration help to restore body fluid homeostasis. The present work shows that MEMRI and ${ }^{1} \mathrm{H}$ MRS 


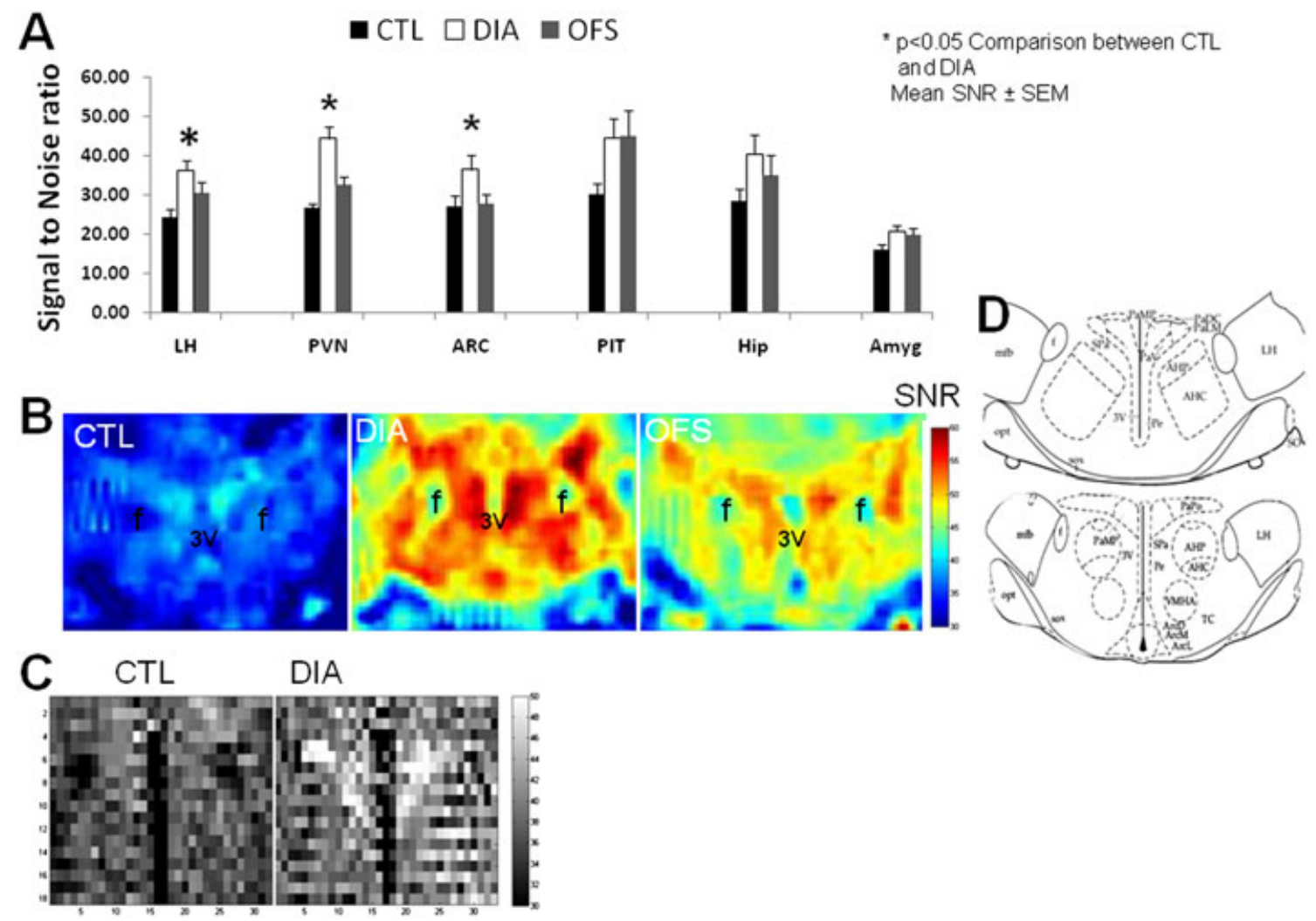

Figure 3. Signal-to-noise ratio (SNR) comparisons. (A) Comparison of SNR calculated in small regions of interest (ROIs) in the lateral hypothalamus $(\mathrm{LH})$, paraventricular nuclei (PVN), arcuate nucleus (ARC), pituitary gland (PIT), hippocampus (Hip) and amygdala (Amyg) of rats (CTL, control; DIA, dehydration-induced anorexia; OFS, overnight food suppression). Data are presented as the mean \pm standard error of the mean (SEM). (B) Example of false color-coded SNR pixel-by-pixel maps in CTL, DIA and OFS rats imaged on the same day. (C) Example of SNR pixel-by-pixel maps in CTL and DIA rats imaged on the same day. (D) Schematic drawing of a coronal slice at a distance of $-1.80 \mathrm{~mm}$ from the bregma copied from the atlas of Paxinos and Watson (22) and corresponding to the slices shown in (B) and (C). An ROI was drawn encompassing several hypothalamic structures, such as the PVN and LH. AHC, central part of the anterior hypothalamic area; AHP, posterior part of the anterior hypothalamic area; ArcD, arcuate nucleus, dorsal part; ArcL, arcuate nucleus, lateral part; ArcM, arcuate nucleus, medial part; f, fornix; mfb, median forebrain bundle; opt, optic tract; PaDC, paraventricular hypothalamic nucleus, dorsal cap; PaLM, anterior magnocellular part of the PVN of the hypothalamus; PaMP, medial parvicellular zone of the PVN of the hypothalamus; PaV, ventral part of the PVN; Pe, PVN of the hypothalamus; SO, supraoptic nucleus; sox, supraoptic decussation; SPa, subparaventricular zone of the hypothalamus; TC, tuber cinereum area; VMHA, ventromedial hypothalamic nucleus, anterior part; $3 \mathrm{~V}$, third ventricle.

are powerful techniques to obtain an understanding of the differences between the physiological, behavioral, neuronal and metabolic responses to fasting and DIA in the hypothalamus.

\section{MEMRI for the investigation of hypothalamic structures}

Many invasive biochemical and histological methods have been used to investigate the hypothalamic-pituitary-thyroid axis (11-14). Confocal microscopy and autoradiography are the methods of choice for the imaging of hypothalamic structures in the rat: immunohistochemical staining for Fos-like immunoreactivity has become a standard technique for the investigation of changes in neuronal activity. Despite their invasiveness, these techniques offer the possibility to examine various nuclei of the hypothalamus with high spatial resolution. The PVN contains $10^{4}$ neurons in a volume of approximately $0.5 \mathrm{~mm}^{3}$ on either side of $3 \mathrm{~V}$ (27). c-Fos techniques allow the precise localization of the specific neuroanatomical structures of populations of neurons that respond to stimulation. Quantification is performed simply by counting the number of neurons labeled for Fos. However, the procedures for Fos staining are not dynamic and neuronal stimulations need to be strong and prolonged before quantifiable Fos expression is reached. Furthermore, not all the activated neurons express the Fos gene, and it is not clear how to define a threshold for c-Fos expression (28). Therefore, the absence of c-Fos expression cannot rule out the presence of neuronal activity (28).

For the first time, three-dimensional $T_{1}$-weighted MEMRI images, in which hypothalamic structures, such as the PVN, can be distinguished, have been obtained. Three-dimensional $T_{1}$-weighted gradient echo imaging appears to be an adequate method for MEMRI studies, especially for the investigation of smaller structures $(1,29,30)$. At $14.1 \mathrm{~T}$, the three-dimensional technique allowed a $98 \times 98-\mu \mathrm{m}^{2}$ in-plane spatial resolution following FASTMAP shimming. Increased $T_{1}$ weighting of threedimensional images, as well as increased spatial resolution, would certainly further improve the detection of specific hypothalamic structures. A recent study (31) has shown that ultrahigh-field micro-imaging of the rodent brain can reveal interesting anatomical structures up to an in-plane resolution of $33 \mu \mathrm{m}$, although the aforementioned study was performed by taking advantage of susceptibility differences at $14.1 \mathrm{~T}$. 
Figure 4. Statistical maps of differential manganese-related signals for rats (CTL, control; DIA, dehydration-induced anorexia; OFS, overnight food suppression). (A) $T$-score maps for CTL versus OFS and OFS versus CTL rats. (B) $T$-score maps for CTL versus DIA and DIA versus CTL rats. (C) $T$-score maps for OFS versus DIA and DIA versus OFS rats. Results are presented as color-coded $T$-scores thresholded using clusters to a significance level of $p<0.05$. Brain regions were identified by overlaying images on the rat brain atlas of Paxinos and Watson (22). (D) Hypothalamic brain structures can be identified by reference to the panel of the atlas of Paxinos and Watson (22) at level 28 (distance from bregma, $-2.12 \mathrm{~mm}$ ). A table of the list of abbreviations is given.

Detection of enhancement in the hypothalamus with MEMRI following overnight food deprivation and dehydration challenges

In this study, increased $\mathrm{MnCl}_{2}$ enhancement was seen in the $\mathrm{LH}$, PVN and ARC of DIA and OFS rats compared with ad libitum-fed rats, in line with previous MEMRI studies (32-34). Compared with previous studies, $\mathrm{Mn}^{2+}$ enhancement was examined at $24 \mathrm{~h}$ postinjection without using dynamic $T_{1}$-weighted methods. Moreover, there was no need to disrupt the blood-brain barrier (35). Using a dynamic $T_{1}$-weighted protocol, Kuo et al. (34) showed significant differences in enhancement between fasted and nonfasted mice that also underwent overnight fasting: PVN, approximately $+10 \%$; ventromedial hypothalamus, approximately $+20 \%$; periventricular nucleus of the hypothalamus, approximately $+24 \%$; ARC, approximately $+20 \%$; however, no significant difference was found in PIT. In the present study, significant SNR differences were found between CTL and OFS rats in hypothalamic regions with small ROI analysis: SNR positive differences of $39 \%$ and $22 \%$ were found in
LH and PVN, respectively, for OFS rats compared with CTL rats. Partial volume effects, as well as variability between animals of the same group, may explain why no statistically significantly different SNRs were found in ARC and PIT. Pixel-by-pixel SNR maps, as well as $T$-scores, revealed increased differences in PVN and $\mathrm{LH}$ and along 3V. In ARC of fasted mice, Kuo et al. (34) detected a fast and strong signal enhancement during $\mathrm{Mn}^{2+}$ infusion, as well as faster signal intensity decay post-infusion, relative to other hypothalamic regions. Therefore, $\mathrm{Mn}^{2+}$ washout at $24 \mathrm{~h}$ post-injection may explain why no significant difference can be seen in ARC in both ROls and pixel-by-pixel maps.

Both $\mathrm{ROI}$ and pixel-by-pixel analyses revealed statistically significant SNR enhancement in DIA rats with respect to CTL and OFS rats. Increased SNR in the hypothalamus of DIA rats relative to CTL rats was measured in PVN (approximately $+59 \%$ ), $\mathrm{LH}$ (approximately $+53 \%$ ) and ARC and PIT (approximately $+28-36 \%$ ). PVN are located inside the blood-brain barrier. Earlier MEMRI studies showed that blood-brain barrier disruption was necessary to detect signal intensity enhancement in these nuclei (35). 
A
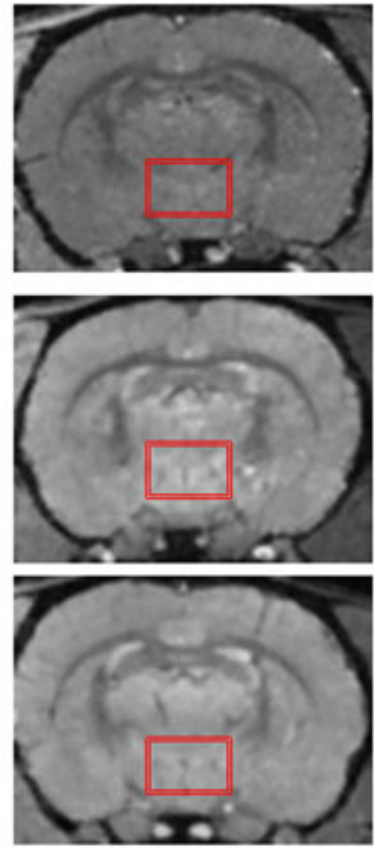

CTL

B
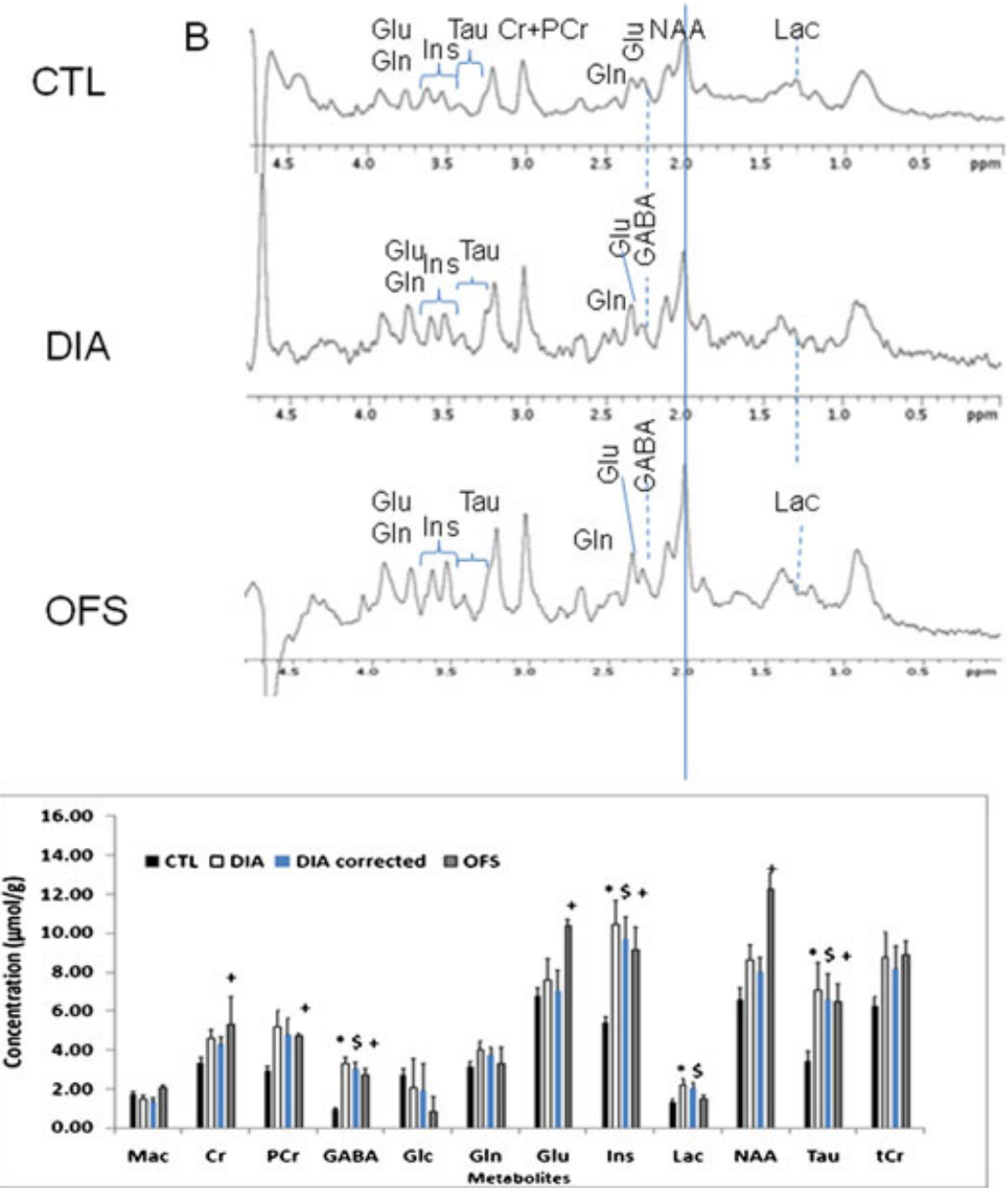

Figure 5. Single-voxel spectra acquired in the hypothalamus of rats at $14.1 \mathrm{~T}$ with metabolite assignments. (A) Example of a voxel of interest (localized in the hypothalamus) overlaid over three-dimensional, $T_{1}$-weighted images of rats (CTL, control; DIA, dehydration-induced anorexia; OFS, overnight food suppression). (B) Spectra of CTL, DIA and OFS rats. (C) Neurochemical profile of the paraventricular nucleus of the lateral hypothalamus acquired at 14.1 T. Metabolites were compared in a voxel of interest of identical size for CTL, DIA and OFS rats. Data are presented as the mean \pm standard error of the mean (SEM). An unpaired two-tailed $t$-test was used; $p<0.05$ was considered to be significant. *Statistically significant difference for CTL versus DIA rats. +Statistically significant difference for CTL versus OFS rats. DIA-corrected represents the neurochemical profile of DIA rats assuming a $10 \%$ loss of brain water. \$Statistically significant difference for CTL versus DIA-corrected rats. Cr, creatine; GABA, $y$-aminobutyric acid; Glc, glucose; Gln, glutamine; Glu, glutamate; Ins, myo-inositol; Lac, lactate; Mac, macromolecules; NAA, N-acetylaspartate; PCr, phosphocreatine; Tau, taurine; tCr, total creatine $(\mathrm{Cr}+\mathrm{PCr})$.

However, recent studies (32-34), including the present work, have shown that $\mathrm{Mn}^{2+}$ slowly distributes to distal regions from ARC where the blood-brain barrier is not present. Increased activation in hypothalamic nuclei and other brain structures as a result of saline administration was also found in previous MEMRI and c-Fos studies (35).

\section{Evaluation of relevant differences between CTL, OFS and DIA rats}

Although it is important to detect $\mathrm{Mn}^{2+}$ enhancement within and between groups of animals, it is also important and challenging to define appropriate methods to compare images of functional information between groups of animals. Techniques currently rely on image normalization and registration processes, whereby images are transformed into the same stereotactic space for subsequent analysis. Recently, such optimized analyses have been conducted in other MEMRI studies $(29,36)$. In the present study, group-averaged T-score maps were used. One of the main results of this analysis was that fasting and DIA significantly affected a large part of the rat brain. Significant changes were revealed not only in the hypothalamus, as expected, but also in the amygdala and septal nuclei, which has not been reported previously for DIA.

One of the major goals of current studies of the regulation of food intake is to determine reproducible and recognizable markers of feeding behavior. In anorexia, the drive to increase food intake in the face of negative energy balance is reduced or totally eliminated. The mechanisms inhibiting appetite and food intake are still poorly understood. In the current study, we aimed to determine the differences in the hypothalamus between fasted and anorexic rats using MEMRI. For a further understanding of anorexigenic mechanisms, it is important to identify specific neuronal activations in specific areas of the hypothalamus. The $T$-score maps revealed significant increases in activation patterns that should correspond to increased neuronal activity within the PVN 
and LH of DIA and OFS with respect to CTL rats. Moreover, increased activation of PVN and LH was found for DIA compared with OFS rats. These findings appear to correlate with previous studies on neuronal activation of the hypothalamus following fasting and DIA: the drinking of hypertonic saline elicited statistically significant Fos expression in the LH, ARC, lateral parvicellular part (autonomic and behavioral control) and lateral magnocellular part (endocrine control) of the PVN of DIA rats compared with the corresponding areas in control rats (11). Acute fasting (24-72 h) also increased Fos expression in several nuclei of the hypothalamus (37). Nevertheless, attributing increased MEMRI enhancement to specific nuclei in the hypothalamus appears to be difficult at this stage and requires further investigation.

Studies investigating an inhibitor of food intake, glucagon-like peptide 1 (GLP1), demonstrated significant increases in $\mathrm{Mn}^{2+}$ enhancement in the PVN and ARC relative to fasted animals (32-34). GLP1 clearly demonstrated an anorectic effect mediated through the PVN of the hypothalamus with a reduction in orexigenic neuron [NPY/Agouti-related protein (AgRP)] expression, an enhancement of anorexigenic neuron [pro-opiomelanocortin/ cocaine-amphetamineregulated transcript (POMC/CART)] expression and a reduction in adenosine-5'-monophosphate-activated kinase (AMP) expression opposed to that seen in 48-h-fasted rats (38). Although DIA may follow similar mechanisms, it appears that, in this model of anorexia, the role of NPY is more complex (14).

\section{Neurochemical profile of the hypothalamus investigated using ${ }^{1} \mathrm{H}$ MRS at $14.1 \mathrm{~T}$}

For a better understanding of the metabolic mechanisms engaged to generate anorexia, the neurochemical profiles of the PVN and LH of the hypothalamus were determined using ${ }^{1} \mathrm{H}$ MRS at $14.1 \mathrm{~T}$. The measurement of the rat hypothalamic neurochemical profile by MRS is not an easy task because of its deep localization within the rat brain. In the present study, a few challenges were overcome by measuring the neurochemical profile of the upper part of the hypothalamus at $14.1 \mathrm{~T}$. A dedicated in-house-built flat quadrature surface coil (ellipse-shaped loops) was centered over the upper part of the hypothalamus of the rat brain and, to enhance power settings, a 500-W radiofrequency amplifier was used. ${ }^{1} \mathrm{H}$ spectroscopy was performed only when the water linewidth was below $28 \mathrm{~Hz}$ for acceptable water suppression and outer volume suppression.

The neurochemical profiles of the PVN and LH were significantly different in DIA and OFS rats relative to ad libitum-fed rats. However, similar neurochemical profiles were measured in DIA and OFS rats.

Osmotic dehydration is caused by salt loading. In response to changes in osmolality, a cell shrinks or swells and then exhibits a regulatory volume increase or decrease. On hypernatremic challenge, a cell loses water until the intracellular and extracellular osmolality are equal. The recovery of water is mediated through inorganic $\left(\mathrm{Na}^{+}, \mathrm{K}^{+}, \mathrm{Cl}^{-}\right)$and organic [Glu, glutamine (GIn), Tau, myo-Ins] osmolytes (26).

Rats (39-41) and rabbits (41) exposed to acute hypernatremia demonstrate a rapid decrease in brain water content (7-12\%), followed by an increase in solute concentration. Volume regulation is mediated by $\mathrm{Na}^{+}, \mathrm{K}^{+}$and $\mathrm{Cl}^{-}$derived from the bulk flow of $\mathrm{NaCl}$ from the cerebrospinal fluid and contributions from blood and transcellular water. Under sustained hypernatremia over several days, brain water and volume values are restored to normal. Moreover, prolonged hypernatremia results in an accumulation of organic osmolytes to restore the total brain water content to normal levels.

In the case of prolonged hypernatremia, as in this study, DIA rats have unrestricted access to hypertonic water. Cellular dehydration is expected to be compensated for by the continuous ingestion of water loaded with sodium over 4 days of the protocol. As a consequence, volume regulation and cerebral adaptation occur. The formation of edema was not observed. In addition, no renal dysfunction was observed in rats, as urine was normally excreted over 4 days and excess sodium should have been rapidly excreted in the urine.

In DIA rats, significant increases in GABA, Glu, $\mathrm{Cr}$ and Tau were found. PCr, NAA and Gln also showed a tendency to increase. Simultaneously, a tendency for glucose to decrease was found, but was not significant. Previous studies in dehydrated anorexic rats have shown that normal plasma glucose concentrations are maintained (42). These observations show that the response to negative energy balance is biased towards increased glycogenolysis and lipolysis, as expected. Similar changes were found in OFS rats, showing that DIA rats maintain a normal metabolic response to reduced food intake.

However, increased levels of Lac were detected in DIA rats, but not OFS rats. A recent study in mice hypothalamus, using ${ }^{13} \mathrm{C}$ highresolution magic angle spinning (HR-MAS), demonstrated Lac accumulation following overnight fasting (43), as well as an increase in GABA. The increase in GABA was attributed to increased synthesis involving an increased activity of the Glu-Gln-GABA cycle, as Glu and Gln are the main precursors of GABA (44). This hypothesis matches the results of the present study, where significant increases in Glu and Gln concentrations were found in both DIA and OFS rats. Levels of Lac rose in DIA rats, following the hypothesis of Lac acting as a neuronal fuel $(44,45)$ as well as the astrocyte to neuron Lac shuttle hypothesis $(44,45)$.

\section{Anorexic behavior and hyperosmolality}

Hyperosmolality has been shown to generate glucose oxidation, Lac production, the release of ammonia and amino acids, and changes in brain nitrogen, sodium and potassium in the rat brain $(46,47)$. GABA concentrations increase significantly in rat brain as a result of $\mathrm{NaCl}(240 \%)$, sucrose $(98 \%)$ and glucose $(26 \%)(46,47)$. Increases in Tau, Glu, aspartate and GIn have been attributed to salt dependence only (46). Interestingly, changes in brain GABA, Glu, aspartate and Tau do not seem to be influenced by their elevation in the plasma (46). However, changes in metabolites are weighted by the degree of hyperosmolar challenge. The protocol used in this study was closest to the chronic moderate hypernatremia model employed by Lien et al. (47). Their results were similar to those observed in our study for DIA rats. Increases in Tau, Glu, Gln, inositol and glycine, known as 'idiogenic osmoles', serve to maintain the cell volume in response to a hypertonic challenge $(46,47)$. This mechanism is expected to avoid the disruption of enzyme function at the cellular level. The genesis of idiogenic osmoles is not regionally specific: increases were noted in both the hypothalamus and hippocampus in our study and for entire brain extracts in other studies $(46,47)$.

In the hypothalamus, changes in the osmoregulation mechanisms demonstrate implications for the regulation of food intake (10). Dehydration mechanisms, such as chronic water deprivation, produce increased NPY expression in the ARC to the same extent as salt loading (48), but have different effects in the PVN (49). Moreover, the hypothalamus plays a fundamental role in the 
maintenance of body fluid homeostasis through vasopressin and oxytocin release in response to osmotic stimuli $(50,51)$. In particular, it has been shown that hyperosmotic stimulation increases oxytocin secretion (15) in the PVN, which receives inputs from the forebrain osmosensitive network to regulate both vasopressin and oxytocin secretion.

The neurochemical profile of the PVN-LH in OFS rats presented similar characteristics to that in DIA rats. In the OFS group, changes in metabolite concentrations cannot be attributed to hyperosmolality. One hypothesis may be that fasting also induces osmotic changes in the hypothalamus in response to the need to maintain plasma osmolality in the absence of food (52).

\section{Importance of GABA}

Fasting and DIA induce similar profiles of caloric deficits in rats (42). Neurons of the PVN synthesize thyrotropin-releasing hormone (TRH) and corticotropin-releasing hormone (CRH) which play important roles in energy homeostasis (11). Under a restricted food regime, rats show decreased TRH expression in the PVN and increased expression of NPY in the ARC (11). By contrast, anorexic animals show increased TRH expression in the PVN. Food-restricted and DIA animals show a similar reduction in CRH expression compared with control animals. In the present work, they showed a similar increase in GABA levels, known to suppress feeding in rats and to have an impact on body weight loss (53). Watts (15) suggested that $\mathrm{CRH}$ neurons of the $\mathrm{LH}$ are specifically activated by the dehydration-induced stimulus and project to TRH neurons in the PVN, so that food inhibition as a result of low energy intake is overridden by dehydration mechanisms. In addition, the majority of inputs to neurons of the PVN contain the inhibitory neurotransmitter GABA. No GABAergic neurons are located within the PVN itself (54). GABAergic projections from regions surrounding the PVN (anterior hypothalamic area, dorsomedial hypothalamic nucleus, LH, medial pre-optic area), as well as from the forebrain, convey inhibitory signals to $\mathrm{CRH}$ neurons in response to a stressor, such as DIA or food deficiency, and limit hypothalamic activity by an important braking mechanism that includes an increase in GABA release at the level of the PVN (54). A potential explanation of the similar GABA levels found in OFS and DIA rats may be the regulation of the hypothalamus via different GABA-A receptor subunits $(55,56)$.

\section{CONCLUSIONS}

Stimulation of the PVN and LH of the hypothalamus on cellular dehydration produces changes in both neurochemistry and neuronal activation. A specific response of the hypothalamicpituitary-thyroid axis is detectable with high-field MEMRI and MRS. The combination of MEMRI and MRS methods shows promise for the investigation of the mechanisms and neural networks responsible for anorexia.

\section{Acknowledgements}

This work was supported by the Centre d'Imagerie Biomédicale (CIBM) of the University of Lausanne (UNIL), Ecole Polytechnique Fédérale de Lausanne (EPFL), University Hospital of Geneva (HUG), Centre Hospitalier Universitaire Vaudois (CHUV), and the Leenards and Jeantet Foundations. The authors wish to thank Dr Lijing Xin and Dr Cristina Cudalbu for their help with image processing and ${ }^{1} \mathrm{H}$ spectroscopy, and for useful discussions.

\section{REFERENCES}

1. Silva AC, Lee JH, Aoki I, Koretsky AP. Manganese-enhanced magnetic resonance imaging (MEMRI): methodological and practical considerations. NMR Biomed. 2004; 17: 532-543.

2. Aoki I, Naruse S, Tanaka C. Manganese-enhanced magnetic resonance imaging (MEMRI) of brain activity and applications to early detection of brain ischemia. NMR Biomed. 2004; 17: 569-580.

3. Pautler RG, Silva AC, Koretsky AP. In vivo neuronal tract tracing using manganese-enhanced magnetic resonance imaging. Magn. Reson. Med. 1998; 40: 740-748.

4. Pautler RG. In vivo, trans-synaptic tract-tracing utilizing manganeseenhanced magnetic resonance imaging (MEMRI). NMR Biomed. 2004; 17: $595-601$

5. Duong TQ, Silva AC, Lee SP, Kim SG. Functional MRI of calciumdependent synaptic activity: cross correlation with CBF and BOLD measurements. Magn. Reson. Med. 2000; 43: 383-392.

6. Doron O, Goelman G. Evidence for asymmetric intra substantia nigra functional connectivity - application to basal ganglia processing. Neuroimage, 2010; 49(4): 2940-2946.

7. Horvath TL. Synaptic plasticity in energy balance regulation. Obesity, 2006; 14: 228S-233S.

8. Pyner S. Neurochemistry of the paraventricular nucleus of the hypothalamus: implications for cardiovascular regulation. J. Chem. Neuroanat. 2009; 38(3): 197-208.

9. Barbarich-Marsteller NC, Marsteller DA, Alexoff DL, Fowler JS, Dewey SL. MicroPET imaging in an animal model of anorexia nervosa. Synapse, 2005; 57(2): 85-90.

10. Berthoud HR. Multiple neural systems controlling food intake and body weight. Neurosci. Biobehav. Rev. 2002; 26(4): 393-428.

11. Jaimes-Hoy $L$, Joseph-Bravo $P$, de Gortari $P$. Differential response of TRHergic neurons of the hypothalamic paraventricular nucleus (PVN) in female animals submitted to food-restriction or dehydration-induced anorexia and cold exposure. Horm. Behav. 2008; 53(2): 366-377.

12. de Gortari $P$, Mancera $K$, Cote-Vélez A, Amaya Ml, Martínez A, Jaimes-Hoy L, Joseph-Bravo P. Involvement of CRH-R2 receptor in eating behavior and in the response of the HPT axis in rats subjected to dehydration-induced anorexia. Psychoneuroendocrinology, 2009; 34(2): 259-272.

13. Salter-Venzon D, Watts AG. The role of hypothalamic ingestive behavior controllers in generating dehydration anorexia: a Fos mapping study. Am. J. Physiol. Regul. Integr. Comp. Physiol. 2008; 295: R1009-R1019.

14. Salter-Venzon D, Watts AG. Site-specific attenuation of food intake but not the latency to eat after hypothalamic injections of neuropeptide $\mathrm{Y}$ in dehydrated-anorexic rats. Am. J. Physiol. Regul. Integr. Comp. Physiol. 2009; 297(6): R1813-R1821.

15. Watts AG. Dehydration-associated anorexia: development and rapid reversal. Physiol. Behav. 1999; 65(4-5): 871-878.

16. Gruetter R. Automatic, localized in-vivo adjustment of all first- and second-order shim coils. Magn. Reson. Med. 1993; 29(6): 804-811.

17. Gruetter R, Tkác I. Field mapping without reference scan using asymmetric echo-planar techniques. Magn. Reson. Med. 2000; 43: 319-323.

18. Tkác I, Henry PG, Andersen P, Keene CD, Low WC, Gruetter R. Highly resolved in vivo ${ }^{1} \mathrm{H}$ NMR spectroscopy of the mouse brain at 9.4T. Magn. Reson. Med. 2004; 52(3): 478-484.

19. Charrier R. Bias Field Correction, Master's Thesis, Ecole Polytechnique Fédérale de Lausanne, Lausanne; 2006.

20. Just N, Kunz N, Charrier R, Bach-Cuadra M, Lei H, Gruetter R, Thiran J-P. Bias field correction of surface coil MR images of rodent brain acquired at high field. Proceedings of the 15th Scientific Meeting ISMRM, Berlin, Germany, 2007; 3713.

21. Styner M, Brechbühler C, Székely G, Gerig G. Parametric estimate of intensity inhomogeneities applied to MRI. IEEE Trans. Med. Imaging, 2000; 19(3): 153-165.

22. Paxinos G, Watson C. The Rat Brain in Stereotaxic Coordinates. Academic Press: San Diego, CA; 1998.

23. Mlynárik V, Gambarota G, Frenkel $H$, Gruetter R. Localized shortecho-time proton MR spectroscopy with full signal-intensity acquisition. Magn. Reson. Med. 2006; 56(5): 965-970.

24. Tkác I, Starcuk Z, Choi IY, Gruetter R. In vivo ${ }^{1}$ H NMR spectroscopy of rat brain at $1 \mathrm{~ms}$ echo time. Magn. Reson. Med. 1999; 41(4): 649-656.

25. Provencher SW. Estimation of metabolite concentrations from localized in-vivo proton MR spectra. Magn. Reson. Med. 1993; 30(6): 672-679. 
26. Sterns RH, Silver SM. Chapter title. In: Alpern RJ, Hebert SC (eds). Seldin and Giebisch's The Kidney: Physiology and Pathophysiology: Hypernatremic States. Academic Press: London; 2007, pp. 1203-1224.

27. Simmons DM, Swanson LW. High-resolution paraventricular nucleus serial section model constructed within a traditional rat brain atlas. Neurosci. Lett. 2008; 438: 85-89.

28. Harris J. Using c-fos as a neural marker of pain. Brain Res. Bull. 1998; 45(1): 1-8.

29. Chuang KH, Lee JH, Silva AC, Belluscio L, Koretsky AP. Manganese enhanced MRI reveals functional circuitry in response to odorant stimuli. Neuroimage, 2009; 44: 363-372.

30. Natt O, Watanabe T, Boretius S, Radulovic J, Frahm J, Michaelis T. High-resolution 3D MRI of mouse brain reveals small cerebral structures in vivo. J. Neurosci. Methods 2002; 120: 203-209.

31. Marques JP, Maddage R, Mlynarik V, Gruetter R. On the origin of the MR image phase contrast: an in vivo MR microscopy study of the rat brain at 14.1 T. Neuroimage, 2009; 46(2): 345-352.

32. Parkinson JR, Chaudhri OB, Kuo YT, Field BCT, Herlihy AH, Dhillo WS, Ghatei MA, Bloom SR, Bell JD. Differential patterns of neuronal activation in the brainstem and hypothalamus following peripheral injection of GLP-1, oxyntomodulin and lithium chloride in mice detected by manganese-enhanced magnetic resonance imaging (MEMRI). Neuroimage, 2009; 44(3): 1022-1031.

33. Chaudhri OB, Parkinson JR, Kuo YT, Druce MR, Herlihy AH, Bell JD, Dhillo WS, Stanley SA, Ghatei MA, Bloom SR. Differential hypothalamic neuronal activation following peripheral injection of GLP-1 and oxyntomodulin in mice detected by manganese-enhanced magnetic resonance imaging. Biochem. Biophys. Res. Commun. 2006; 350: 298-306.

34. Kuo YT, Herlihy AH, So PW, Bell JD. Manganese-enhanced magnetic resonance imaging (MEMRI) without compromise of the bloodbrain barrier detects hypothalamic neuronal activity in vivo. NMR Biomed. 2006; 19(8): 1028-1034.

35. Morita H, Ogino T, Seo Y, Fujiki N, Tanaka K, Takamata A, Nakamura S, Murakami M. Detection of hypothalamic activation by manganese ion contrasted $\mathrm{T}(1)$-weighted magnetic resonance imaging in rats. Neurosci. Lett. 2002; 326(2): 101-104.

36. Zeeni N, Nadkarni N, Bell JD, Even PC, Fromentin G, Tome D, Darcel N. Peripherally injected cholecystokinin-induced neuronal activation is modified by dietary composition in mice. Neuroimage, 2010; 50(4): $1560-1565$.

37. Angeles-Castellanos M, Aguilar-Roblero R, Escobar C. c-Fos expression in hypothalamic nuclei of food-entrained rats. Am. J. Physiol. Regul. Integr. Comp. Physiol. 2004; 286(1): R158-R165.

38. Seo S, Ju S, Chung H, Lee D, Park S. Acute effects of glucagon-like peptide- 1 on hypothalamic neuropeptide and AMP activated kinase expression in fasted rats. Endocrinol. J. 2008; 55(5): 867-874.

39. Cserr HF, De Pasquale M, Patlak CS. Regulation of brain water and electrolytes during acute hyperosmolality in rats. Am. J. Physiol. 1987; 253: F522-F529.

40. Cserr HF, De Pasquale M, Patlak CS. Volume regulatory influx of electrolytes from plasma to brain during acute hyperosmolality. Am. J. Physiol. 1987; 253: F530-F537.
41. Ayus JC, Armstrong DL, Arieff Al. Effects of hypernatremia in the central nervous system and its therapy in rats and rabbits. J. Physiol. 1996; 492: 243-255.

42. Watts AG, Salter DS, Neuner CM. Neural network interactions and ingestive behavior control during anorexia. Physiol. Behav. 2007; 91(4): 389-396.

43. Violante IR, Anastasovska J, Sanchez-Canon GJ, Rodrigues TB, Righi V, Nieto-Charques L, Parkinson JR, Bloom SR, Bell JD, Cerdán S. Cerebral activation by fasting induces lactate accumulation in the hypothalamus. Magn. Reson. Med. 2009; 62(2): 279-283.

44. Pellerin L, Bouzier-Sore AK, Aubert A, Serres S, Merle M, Costalat R, Magistretti PJ. Activity-dependent regulation of energy metabolism by astrocytes: an update. Glia, 2007; 55(12): 1251-1262.

45. Pellerin L, Magistretti PJ. Glutamate uptake into astrocytes stimulates aerobic glycolysis: a mechanism coupling neuronal activity to glucose utilization. Proc. Natl. Acad. Sci. USA, 1994; 91(22): 10 625-10 629.

46. Chan PH, Fishman R. Elevation of rat brain amino acids, ammonia and idiogenic osmoles induced by hyperosmololality. Brain Res. 1979; 161: 293-301.

47. Lien YHH, Shapiro Jl, Chan L. Effects of hypertranemia on organic brain osmoles. J. Clin. Invest. 1990; 85: 1427-1435.

48. O'Shea RD, Gundlach AL. NPY mRNA and peptide immunoreactivity in the arcuate nucleus are increased by osmotic stimuli: correlation with dehydration anorexia. Peptides, 1995; 16(6): 1117-1125.

49. Young WS. Corticotropin-releasing factor mRNA in the hypothalamus is affected differently by drinking saline and by dehydration. FEBS Lett. 1986; 208(1): 158-162.

50. Gomes DA, Giusti-Paiva A, Ventura RR, Elias LL, Cunha FQ, Antunes-Rodrigues J. Carbon monoxide and nitric oxide modulate hyperosmolality-induced oxytocin secretion by the hypothalamus in vitro. Biosci. Rep. 2010; 30(5): 351-357.

51. Ugrumov MV. Magnocellular vasopressin system in ontogenesis: development and regulation. Microsci. Res. Technol. 2002; 56(2): 164-171.

52. Bernal A, Mahia J, Puerto A. Oxytocin, water intake, and food sodium availability in male rats. Horm. Behav. 2007; 52(3): 289-296.

53. Turenius $\mathrm{Cl}$, Htut MM, Prodon DA, Ebersole PL, Ngo PT, Lara RN, Wilczynski JL, Stanley BG. GABA (A) receptors in the lateral hypothalamus as mediators of satiety and body weight regulation. Brain Res. 2009; 1262: 16-24.

54. Cullinan WE, Ziegler DR, Herman JP. Functional role of local GABAergic influences on the HPA axis. Brain Struct. Funct. 2008; 213(1-2): 63-72.

55. Bäckberg M, Ultenius C, Fritschy JM, Meister B. Cellular localization of GABA receptor alpha subunit immunoreactivity in the rat hypothalamus: relationship with neurones containing orexigenic or anorexigenic peptides. J. Neuroendocrinol. 2004; 16(7): 589-604.

56. Mikkelsen JD, Bundzikova J, Larsen MH, Hansen HH, Kiss A. GABA regulates the rat hypothalamic-pituitary-adrenocortical axis via different GABA-A receptor alpha-subtypes. Ann. NY Acad. Sci. 2008; 1148: $384-392$. 